\title{
MRI Safety Update 2008: Part 2, Screening Patients for MRI
}

Frank G. Shellock ${ }^{1}$ Alberto Spinazzi ${ }^{2}$
Keywords: electromagnetic fields, implants, MRI environment, MR conditional, MR safe, MR unsafe, radiology department policies

\section{DOI:10.2214/AJR.08.1038.2}

Received April 2, 2008; accepted after revision May 10, 2008.

F. G. Shellock has received unrestricted educational and research support from Bayer Healthcare; Bracco Diagnostics; Siemens Medical Solutions; Philips Healthcare; GE Healthcare; Toshiba Medical Systems; Hitachi Medical Systems; C.R. Bard; Boston Scientific Corporation; Abbott Laboratories; Medtronic, Inc.; Johnson \& Johnson; St. Jude Medical; Biomet; Lumasense; Advanced Neuromodulation Systems; Arrow International; Smiths Medical; Stryker Instruments; Cordis; DePuy; Integra Neuroscience; Edwards Laboratories; Newmatic Medical; Resonance Technology; Codman; Cyberonics; Smith and Nephew; Inrad; eV3; Cook, Inc.; Stryker; Conor Medical; and Advanced Bionics. A. Spinazzi is an employee of Bracco Diagnostics, Inc.

${ }^{1}$ Keck School of Medicine, University of Southern California and Institute for Magnetic Resonance Safety, Education, and Research, 7511 McConnell Ave., Los Angeles, CA 90045. Address correspondence to F. G. Shellock (frank.shellock@gte.net).

${ }^{2}$ Group Medical and Regulatory Affairs, Bracco Diagnostics, Inc., Princeton, NJ.

\section{AJR2008; 191:1-10}

0361-803X/08/1914-1

(c) American Roentgen Ray Society

OBJECTIVE. This article is the second part of a two-part series on MRI safety. In this article, part 2, the topic of screening patients for MRI procedures is addressed.

CONCLUSION. To prevent incidents and accidents associated with MRI, it is necessary to regularly revisit the safety topics that directly impact patient management especially with respect to the subjects that are "new," those that should be reassessed because of recent changes, topics that deserve emphasis because of controversy or confusion, and information that should be considered in light of new findings.

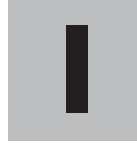
$\mathrm{n}$ consideration of the constant evolution of issues related to MRI safety and the need to update and revise existing guidelines and policies and procedures, there is an ongoing challenge to be aware of the latest developments associated with this topic. Notably, comprehensive reviews and textbooks have been written on the subject of MRI safety and there are Websites with content that is updated on a regular basis [1-15]. Therefore, the reader is referred to those important resources. The goal of this article is to provide an MRI safety update that covers selected topics including those that are "new" (e.g., MRI contrast agents and nephrogenic systemic fibrosis [NSF]), subjects that should be reassessed because of recent changes (e.g., screening patients and individuals), topics that deserve emphasis because of controversy or confusion (e.g., certain policies and procedures), and information that should be considered in light of new findings (e.g., MRI test results for implants and devices, including items evaluated at $3 \mathrm{~T}$ ).

\section{Screening Patients for MRI Procedures and Individuals for the MRI Environment}

The implementation of appropriate policies and procedures to screen a patient for an MRI examination or an individual before permitting entry into the MRI environment (i.e., the MR system room) is a vital aspect of a facility's MRI safety program that, when conducted properly, prevents problems, accidents, and injuries. Therefore, although this topic has been covered in great detail [1, 2, 7-9, 11-17], it needs to be revisited because of the previously described concerns related to the use of MRI contrast agents and NSF as well as other matters that must be considered.

A health care worker specially trained in MRI safety must perform the MRI screening activities whether it involves a patient or another individual. This worker is someone who has undergone training and education to fully understand the potential hazards and issues associated with the MRI environment and MRI procedures and who is aware of the latest information on the screening forms for patients and individuals.

Screening requires the use of written forms [1, 2, 7-9, 11-17]. Two different screening forms, one for patients and one for other individuals, that provide questions to identify potential problems relative to an MRI procedure or the MRI environment have been developed [1, 12, 14, 17]. Screening forms with the latest information are shown in Figure 1, including screening forms for patients in English (Figs. 1A and 1B) and Spanish (Figs. $1 \mathrm{C}$ and $1 \mathrm{D}$ ) and a screening form for nonpatient individuals (Fig. 1E). The form intended for patients includes questions to address NSF risks $[12,14]$. All forms may be downloaded for use at www.MRIsafety.com

Typically, screening forms were created with patients in mind and, therefore, ask certain questions that are inappropriate for individuals who may simply need to enter and work in the MRI environment. Therefore, a 


\section{Shellock and Spinazzi}

separate screening form was created specifically for individuals who need to enter the MRI environment or MR system room. All nonpatient individuals (e.g., MRI technologist, physician, relative, visitor, allied health professional, maintenance worker, custodial worker, fire fighter, security officer) are required to undergo screening before being permitted in the MRI environment. Once the form has been completed, the MRI safetytrained health care worker must review the information and perform a verbal interview to verify the form's content and to allow discussion of any questions or concerns [1, 12, 14, 15-17]. If the individual undergoing screening needs to enter the bore of the MR system and, thus, becomes exposed to the MRI-related electromagnetic fields, this person must be screened using the same form and criteria applied to patients $[12,14]$.

For patient screening, the process may be initiated when scheduling the examination, although this may not be possible in certain cases; at that time, it may be possible to determine whether the patient has an implant that may be potentially contraindicated or that requires special attention for the MRI procedure (e.g., a ferromagnetic aneurysm clip, pacemaker, neurostimulation system) or if the patient has an underlying condition that needs further consideration (e.g., the patient is pregnant, has a disability, has a history of renal failure, has a metallic foreign body). Preliminary screening helps to prevent scheduling patients who may be inappropriate candidates for MRI.

At the MRI center, the patient must undergo comprehensive screening in preparation for the MRI examination. This preparation entails the use of the screening form to facilitate and to document the procedure, a review of the information on the screening form, and a verbal interview to verify the information and allow discussion of any questions or concerns that the patient may have. The MRI safety-trained health care worker must conduct these critical aspects of patient screening. Additional detailed instructions for patient screening have been described previously [1, 2, 7-9, 11-17].

Importantly, both the screening form designed for the patient and the one for other individuals have the following statement to emphasize the need to prevent the introduction of unwanted items into the MR system room:

Remove all metallic objects before entering the MR environment or MR system room including hearing aids, beeper, cell telephone, keys, eyeglasses, hair pins, barrettes, jewelry (including body piercing jewelry), watch, safety pins, paperclips, money clip, credit cards, bank cards, magnetic strip cards, coins, pens, pocket knife, nail clipper, steel-toed boots/shoes, and tools. Loose metallic objects are especially prohibited in the

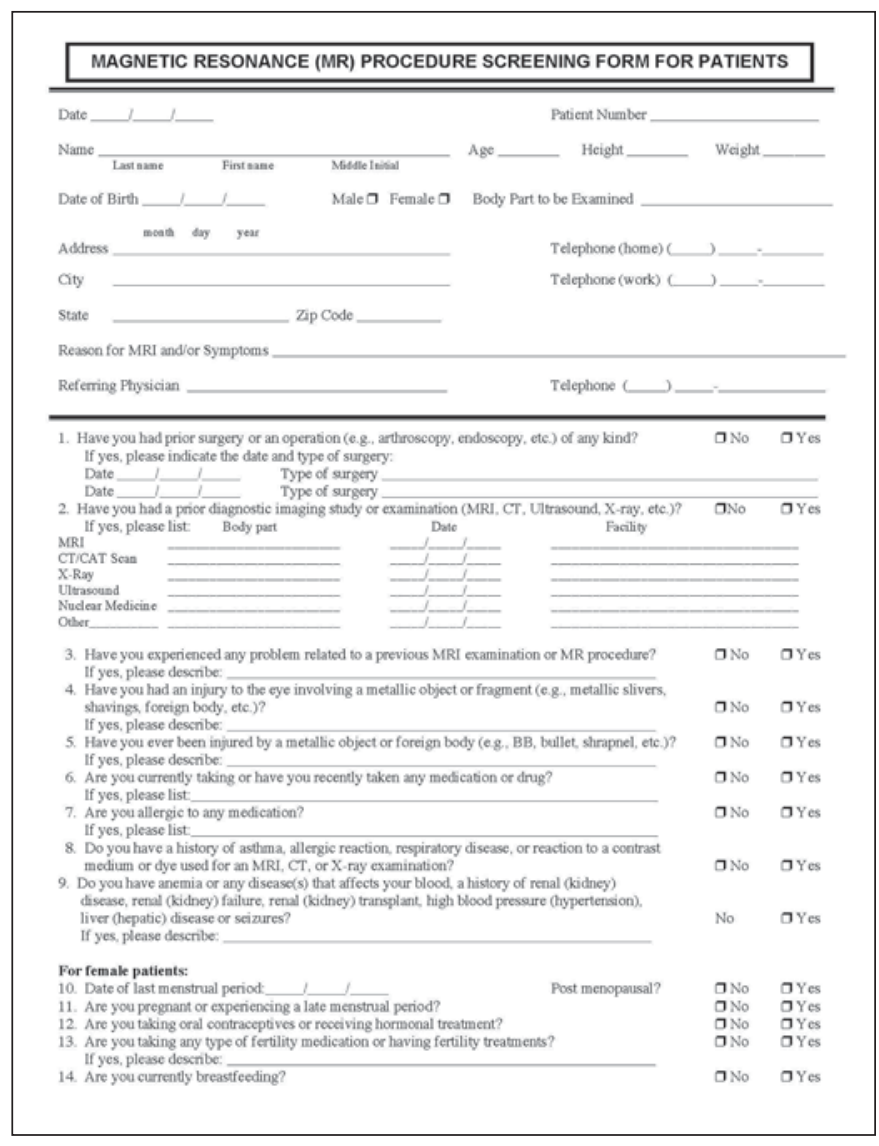

A
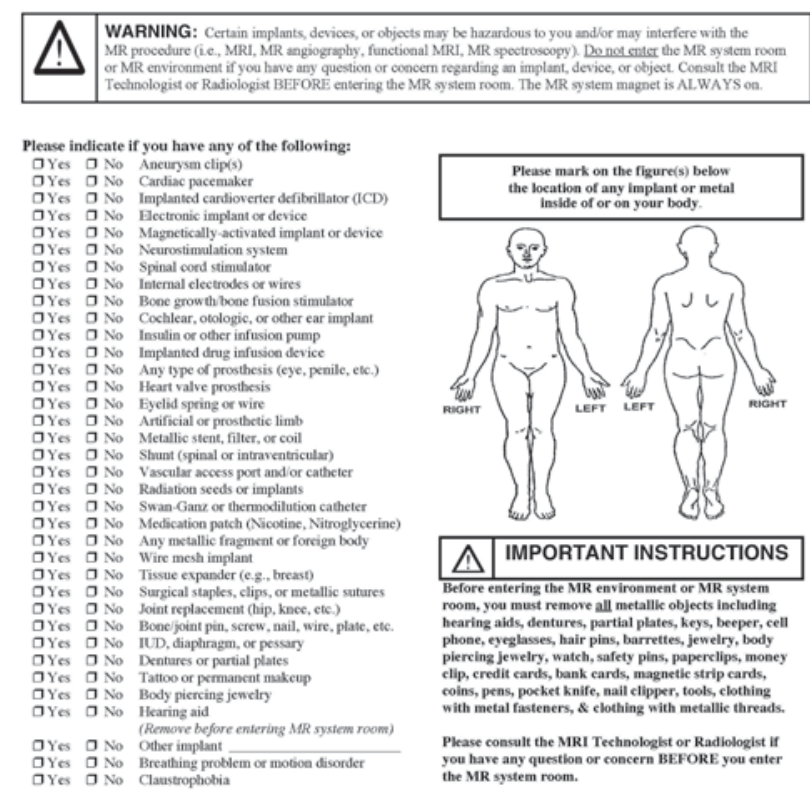

room, you must remore all metallic objects including hearing aids, dentures, partial plates, keys, beeper, cell
phone, eyceglasses, hair pins, barrettes, jewelry, body piercing jeneriry, watch, safely pins, paperclips, money
clip, eredit cards, bank cards, magnetic strip cards, cins, pens, pocket knife, nail clipper, tools, clothing with metal fasteners, \& clothing with metallitic threads. Please consult the MRI Technologitt or Radiologist if
you have any questlon or concern BEFORE you enter the MR system room

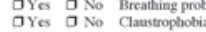
NOTE: You may be advised or required to wear earplugs or other hearing protection during I attest that the above information is correct to the best of my knowledge. I read and understand the contents of this form and had the opportunity to ask questions reganding the information on this form and regarding the MR procedure that I am about to underga. Signamure of Person Completing Form:

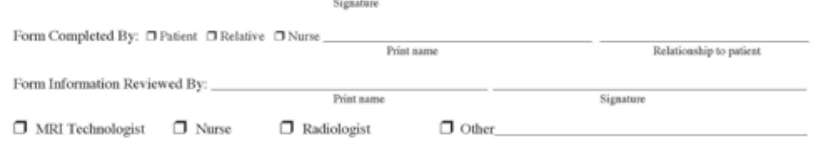

$\square$ MRI Technologist a Nurse a Radiologist a Other

Fig. 1-Forms with latest information used for screening patients before undergoing MRI procedures and for screening nonpatient individuals before entering MRI environment (e.g., MRI technologist, physician, relative, visitor, allied health professional, maintenance worker, custodial worker, fire fighter, security officer). Forms may be downloaded for use at www.MRIsafety.com

A-D, Forms given to patients for screening before MRI ( $A$ and $\mathbf{B}$, in English; $\mathbf{C}$ and $\mathbf{D}$, in Spanish)

(Fig. 1 continues on next page) 


\section{Screening Patients for MRI}

MR system room and MR environment. Please consult the MRI Technologist or Radiologist if you have any questions or concerns BEFORE you enter the MR system room.

This reminder along with standard policies and procedures to control access to the MRI system room and to inspect the patient or individual as well as all items intended for use in the scanner room will serve to prevent accidents and injuries [1, 2, 7-9, 11-17].

\section{Implants and Devices}

An important feature of MRI safety entails the identification of implants and devices and careful consideration of the associated risks [1, 11-17]. Currently, more than 1,800 objects have been tested relative to the use of MRI, with over 600 items evaluated at $3 \mathrm{~T}$ or higher $[12,14]$. MRI test findings at $3 \mathrm{~T}$ are particularly important for patient management given the large increase in clinical applications and growing use of this high-field-strength MR system [18, 19]. Notably, comprehensive reviews discussing MRI information for cardiovascular implants [20], specifically for pacemakers and implantable cardioverter defibrillators [21], have been published recently in the peerreviewed literature. Information for implants and devices is available as a compiled list and in its entirety in an online format at www.MRIsafety.com [12, 14].

New implants and devices are developed on an ongoing basis, which necessitates continuous endeavors to obtain current documentation for these items before subjecting a patient or individual to the MRI environment. In addition, the nuances of MRI testing, especially with respect to evaluating MRI-related heating and identifying functional alterations (which have been described previously [1, 2, 12-14, 22-24]), and the ter- minology applied to label implants and devices must be understood to facilitate patient management [12, 24, 25]. Importantly, for electronically activated implants, the labeling that ensures the safe use of MRI is highly specific to the conditions that were used to assess the device and any deviation from the defined procedures can lead to deleterious effects, severe patient injuries, or fatalities [1, 13-17, 20-23].

\section{Terminology}

A recent "Sentinel Alert" from the Joint Commission [26] states the following:

In general, do not bring any device or equipment into the MRI environment unless it is proven to be MR Safe or MR Conditional. MR Safe items pose no known hazard in all MRI environments, and MR Conditional items have been demonstrated to pose no known hazards in a specified MRI environment with
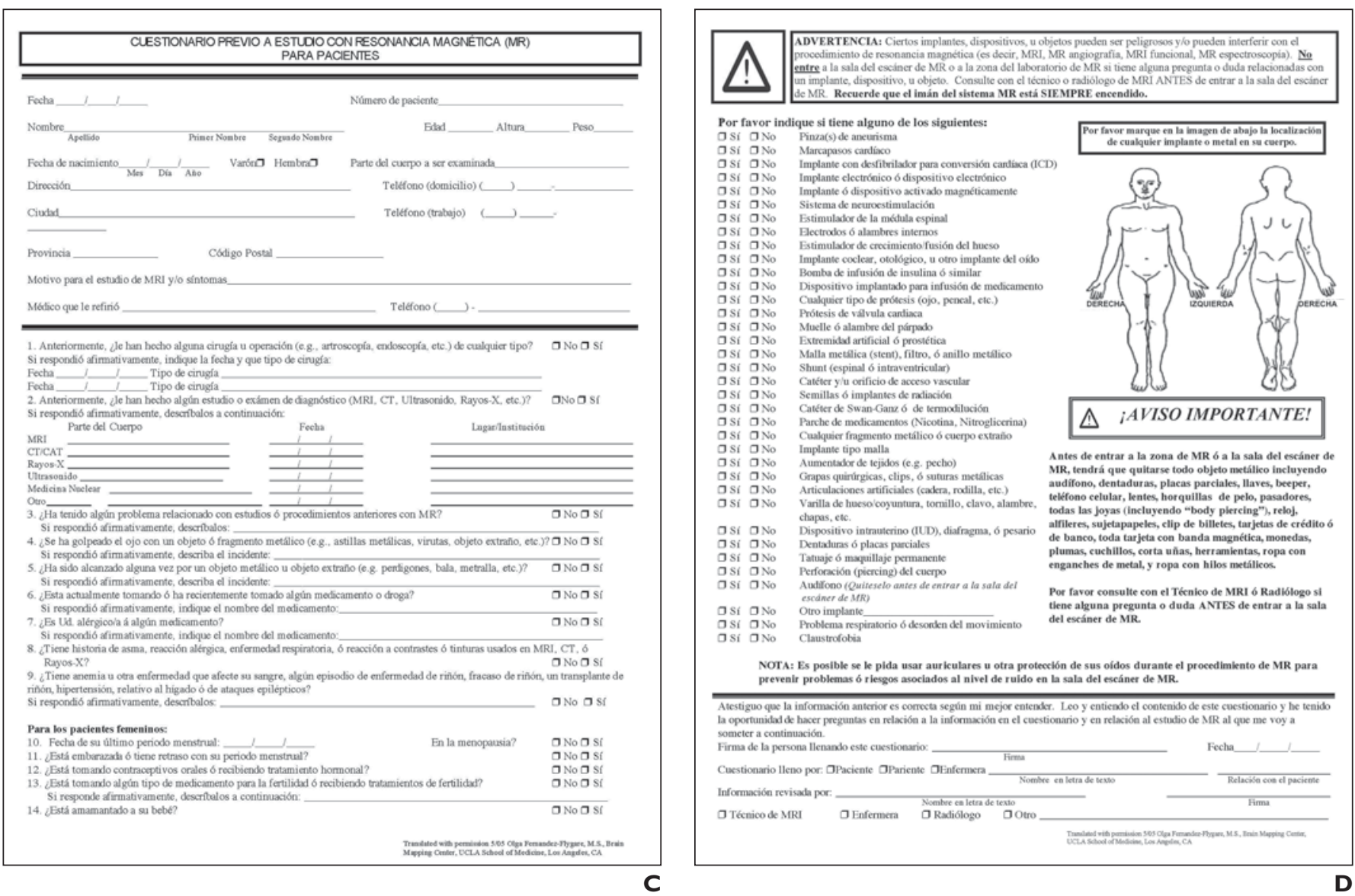

Fig. 1 (continued)—Forms with latest information used for screening patients before undergoing MRI procedures and for screening nonpatient individuals before entering MRI environment (e.g., MRI technologist, physician, relative, visitor, allied health professional, maintenance worker, custodial worker, fire fighter, security officer). Forms may be downloaded for use at www.MRIsafety.com.

A-D, Forms given to patients for screening before MRI (A and B, in English; $\mathbf{C}$ and $\mathbf{D}$, in Spanish).

(Fig. 1 continues on next page) 


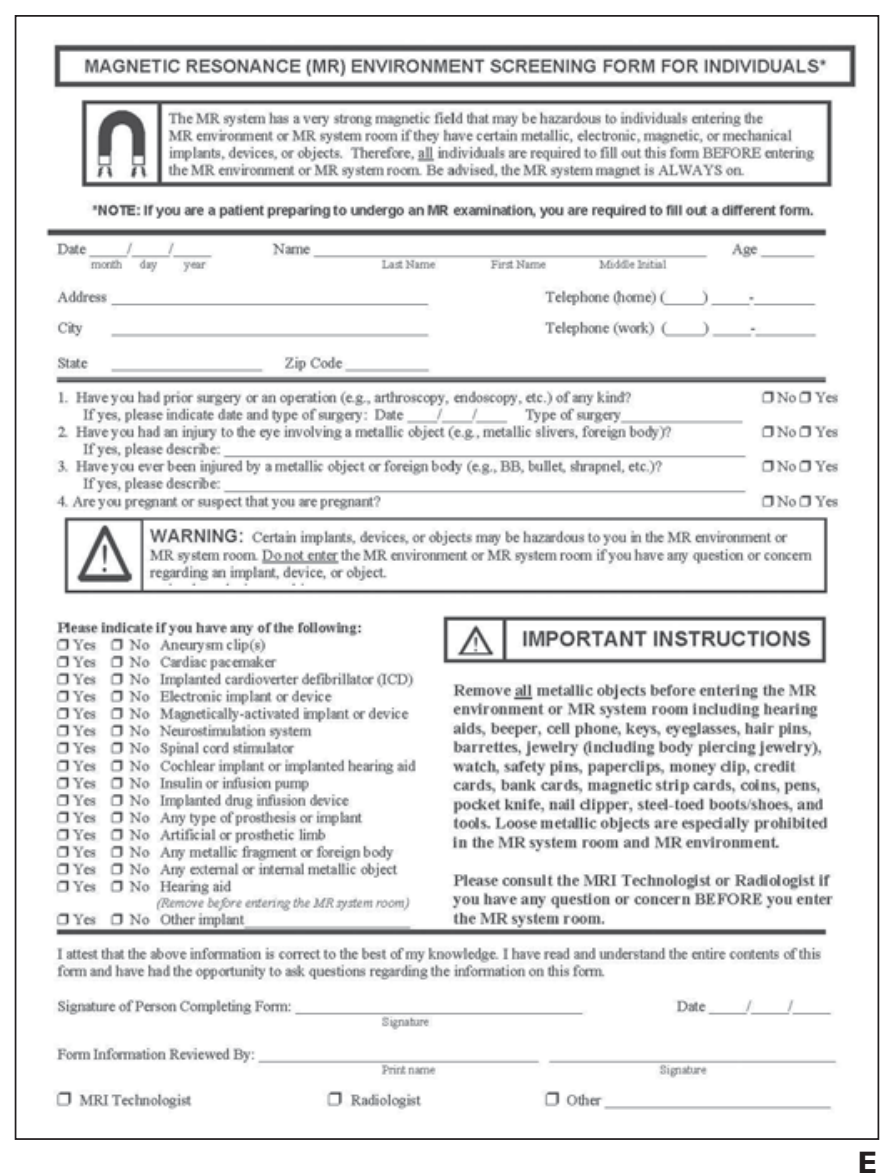

Fig. 1 (continued)-Forms with latest information used for screening patients before undergoing MRI procedures and for screening nonpatient individuals before entering MRI environment (e.g., MRI technologist physician, relative, visitor, allied health professional, maintenance worker, custodial worker, fire fighter, security officer). Forms may be downloaded for use at www.MRIsafety.com.

E, Form used to screen individuals prior to entry into MRI environment.

specified conditions of use. The safety of "MR conditional" items must be verified with the specific scanner and MR environment in which they will be used.

Importantly, this statement refers to terminology that has only been used for labeling of implants and devices since approximately August 2005 [12, 14, 24, 25] and fails to recognize that these terms have not been applied retrospectively by the U.S. Food and Drug Administration (FDA) [12, 14].

The terminology applied to implants and devices relative to the MRI environment has evolved over the years. In 1997, the Center for Devices and Radiological Health proposed definitions for the terms "MR safe" and "MR compatible" as follows [27]:

MR safe: The device, when used in the MRI environment, has been demonstrated to present no additional risk to the patient or other individual, but may affect the quality of the diagnostic information. The MRI conditions in which the device was tested should be specified in conjunction with the term MR safe since a device which is safe under one set of conditions may not be found to be so under more extreme MRI conditions.

MR compatible: A device shall be considered "MR compatible" if it is MR safe and the device, when used in the MRI environment, has been demonstrated to neither significantly affect the quality of the diagnostic information nor have its operations affected by the MR system. The MRI conditions in which the device was tested should be specified in conjunction with the term MR safe since a device which is safe under one set of conditions may not be found to be so under more extreme MR conditions.
To implement this terminology, "MR safety" testing of an implant or object involved assessments of magnetic field interactions, heating, and, in some cases, induced electrical currents, whereas "MR compatibility" testing required all of these as well as characterization of artifacts. In addition, it may have been necessary to evaluate the impact of various MRI conditions on the functional or operational aspects of an implant or device $[12,14]$.

Thus, over the years, manufacturers generally used the terms "MR safe" and "MR compatible" when labeling medical implants and devices. However, in time it became apparent that these terms were confusing and were often used interchangeably or incorrectly [28]. Therefore, in an effort to clarify the terminology and, more importantly, because the misuse of these terms could result in serious accidents for patients and other individuals, the MR Task Group of the American Society for Testing and Materials (ASTM) International developed a new set of terms with associated icons [24, 25]. The new terms-MR safe, MR conditional, and MR unsafe-are defined in an ASTM International document [25] as discussed below.

MR safe-An item that poses no known hazards in all MRI environments. Using the new terminology, "MR safe" items include nonconducting, nonmetallic, nonmagnetic items such as a plastic Petri dish. An item may be determined to be MR safe by providing a scientifically based rationale rather than test data.

$M R$ conditional-An item that has been demonstrated to pose no known hazards in a specified MRI environment with specified conditions of use. Field conditions that define the MRI environment include static magnetic field strength, spatial gradient, time-varying magnetic field $(d B / d t)$, radiofrequency (RF) fields, and specific absorption rate (SAR). Additional conditions, including specific configurations of the item (e.g., the routing of leads used for a neurostimulation system), may be required.

For MR conditional items, the item labeling includes results of testing sufficient to characterize the behavior of the item in the MRI environment. In particular, testing for items that may be placed in the MRI environment should address magnetically induced displacement force and torque and RF heating. Other possible safety issues include but are not limited to thermal injury, induced currents and voltages, electromagnetic 


\section{Screening Patients for MRI}

compatibility, neurostimulation, acoustic noise, interaction among devices, and the safe functioning of the item and the safe operation of the MR system. Any parameter that affects the safety of the item should be listed, and any condition that is known to produce an unsafe condition must be described.

MR unsafe-An MR unsafe item is one that is known to pose hazards in all MRI environments. MR unsafe items include magnetic items such as a pair of ferromagnetic scissors.

In addition to the new terms, ASTM International introduced corresponding icons consistent with international standards for colors and shapes of safety signs [24, 25]. The icons are intended for use on items that may be brought into or near the MRI environment as well as in product labeling. The icons may be reproduced in color or in black and white; however, the use of color is encouraged because of the added visibility.

The MR safe icon consists of the letters "MR" in green in a white square with a green border or the letters "MR" in white within a green square. The MR conditional icon consists of the letters "MR" in black inside a yellow triangle with a black border. The MR unsafe icon consists of the letters "MR" in black on a white field inside a red circle with a diagonal red band. For MR conditional items, the item labeling must include results of testing sufficient to characterize the behavior of the item in the MRI environment.

The new terminology is intended to help clarify matters related to biomedical implants and devices to ensure the safe use of MRI technology. Importantly, as previously indicated, this new terminology has not been applied retrospectively to implants and devices that previously received FDA-approved labeling using the terms "MR safe" or "MR compatible." Accordingly, this should be understood to avoid undue confusion regarding the matter of labeling for "older" versus "newer" implants. To date, relatively few implants have the term "MR conditional" applied in comparison with those labeled using the previous labeling scheme, MR safe and MR compatible [12, 14].

\section{Information for New or Controversial Implants and Devices}

Although a summary of the MRI test data that are available for the more than 1,800 implants and devices is not within the scope of this article, a selection of items evaluated in the MRI environment is presented to illustrate new or controversial findings for these objects, with an emphasis on electronically activated implants as well as 3-T information.

\section{ActiPatch}

The ActiPatch (BioElectronics) is a medical drug-free device that delivers pulsed electromagnetic frequency therapies to accelerate healing of soft-tissue injuries. The ActiPatch has an embedded battery-operated microchip that delivers continuous pulsed therapy to reduce pain and swelling.

The ActiPatch must be removed before a patient undergoes an MRI procedure to prevent possible damage to this device and the potential risk of excessive heating.

\section{Body-Piercing Jewelry}

Ritual or decorative body piercing is popular as a form of self-expression. Different types of materials are used to make bodypiercing jewelry including ferromagnetic and nonferromagnetic metals as well as nonmetallic materials [12, 14, 29-31]. The presence of body-piercing jewelry made from ferromagnetic material presents potential problems for a patient referred for an MRI examination or an individual in the MRI environment. Risks include uncomfortable sensations from movement or displacement that may be mild to moderate depending on the site of the body piercing and the ferromagnetic qualities of the jewelry (e.g., mass, degree of magnetic susceptibility). In extreme cases, serious injuries may occur. In addition, for body-piercing jewelry made from electrically conducting material forming certain lengths or closed loops of a certain diameter, there is the possibility of MRI-related heating that could cause excessive temperature increases and burns $[12,14]$.

Because of safety issues, metallic bodypiercing jewelry should be removed before entering the MRI environment. However, patients or individuals with body piercing are frequently reluctant to remove their jewelry. Therefore, if it is not possible to remove metallic body-piercing jewelry, the patient or individual should be informed regarding the potential risks. In addition, if the bodypiercing jewelry is made from ferromagnetic material, some means of stabilization (e.g., application of adhesive tape or bandage) should be used to prevent movement or displacement. To prevent MRI-related heating of body-piercing jewelry made from conductive materials (e.g., the pierced body part will be in the area of the transmit RF coil), gauze, tape, or other similar material should be used to wrap the jewelry in such a manner as to insulate it (i.e., prevent contact) as much as possible from the underlying skin $[12,14]$. The patient should be instructed to immediately inform the MR system operator if any heating or other unusual sensation is felt in association with the body-piercing jewelry. If this occurs, scanning should be stopped immediately and steps should be taken to prevent possible patient injury.

\section{Foley Catheter with Temperature Sensor}

MRI safety instructions-The MRI safety instructions [14] for the Bardex Latex-Free Temperature-Sensing 400-Series Foley Catheter (C.R. Bard, Inc.) include the following warning:

This product should never be connected to the temperature monitor or connected to a cable during an MRI procedure. Failure to follow this guideline may result in serious injury to the patient. Refer to "Instructions for Use." It is important to closely follow these specific conditions that have been determined to permit the examination to be conducted safely. Any deviation may result in a serious injury to the patient.

Nonclinical testing showed that these Foley catheters with temperature sensors are MR conditional. A patient with one of these devices can be scanned safely immediately after placement under the following conditions:

- static magnetic field of $3 \mathrm{~T}$ or less with regard to magnetic field interactions,

- spatial gradient magnetic field of $720 \mathrm{G} /$ $\mathrm{cm}$ or less with regard to magnetic field interactions, and

- maximum MR system-reported wholebody-averaged SAR of $3.5 \mathrm{~W} / \mathrm{kg}$ at $1.5 \mathrm{~T}$ or 3 $\mathrm{W} / \mathrm{kg}$ at $3 \mathrm{~T}$ for 15 minutes of scanning.

Importantly, the MRI procedure should be performed using an MR system operating at a static magnetic field strength of 1.5 or $3 \mathrm{~T}$ only. The safe use of an MR system operating at a lower or higher field strength for a patient with a Foley catheter with temperature sensor has not been determined.

Special instructions-The position of the wire of the Foley catheter with temperature sensor has an important effect on the amount of heating that may develop during an MRI procedure. Accordingly, the Foley catheter with temperature sensor must be positioned 


\section{Shellock and Spinazzi}

in a straight configuration down the center of the patient table (i.e., down the center of the MR system without any loop) to prevent possible excessive heating associated with an MRI procedure.

Additional MRI safety instructionsAdditional safety instructions include the following:

(1) The Foley catheter with temperature sensor should not be connected to the temperature-monitoring equipment during the MRI procedure.

(2) If the Foley catheter with temperature sensor has a removable catheter connector cable, it should be disconnected before the MRI procedure.

(3) Remove all electrically conductive material from the bore of the MR system that is not required for the procedure (e.g., unused surface coils, cables).

(4) Keep electrically conductive material that must remain in the bore of the MR system from directly contacting the patient by placing thermal and electrical insulation (including air) between the conductive material and the patient.

(5) Position the Foley catheter with temperature sensor in a straight configuration down the center of the patient table to prevent cross points and conductive coils or loops.

(6) The wire and connector of the Foley catheter with temperature sensor should not be in contact with the patient during the MRI procedure; position the device accordingly.

(7) MRI should be performed using an MR system with static magnetic strength of 1.5 or $3 \mathrm{~T}$ only.

(8) At $1.5 \mathrm{~T}$, the MR system whole-bodyaveraged SAR should not exceed $3.5 \mathrm{~W} /$ $\mathrm{kg}$ for 15 minutes of scanning.

(9) At $3 \mathrm{~T}$, the MR system reported wholebody-averaged SAR should not exceed 3 $\mathrm{W} / \mathrm{kg}$ for 15 minutes of scanning.

\section{Glaucoma Drainage Implants (Shunt Tubes)}

A glaucoma drainage implant or device, also known as a shunt tube, is implanted to maintain an artificial drainage pathway to control intraocular pressure for patients with glaucoma [12, 14, 32-37]. Intraocular pressure is lowered when aqueous humor flows from inside the eye through the tube into the space between the plate that rests on the scleral surface and surrounding fibrous capsule. The implantation of a glaucoma drainage device is used to treat glaucoma that is refractory to medical and standard surgical therapy. These cases are usually in patients in whom standard drainage procedures have failed or in patients who have a poor prognosis including failed trabeculectomy, buphthalmos and juvenile glaucoma, neovascular glaucoma and glaucoma secondary to uveitis, traumatic glaucoma, cataract with glaucoma, and high-risk cases of primary glaucoma.

Importantly, for certain glaucoma drainage implants, radiographic findings may suggest the diagnosis of an orbital foreign body if the ophthalmic history is unknown, as reported by Ceballos and Parrish [32]. In that case report, a patient was denied an MRI examination for fear of dislodging an apparent "metallic foreign body." In fact, the patient had a Baerveldt glaucoma drainage implant that was mistakenly identified as an orbital metallic object based on its radiographic characteristics (i.e., due to the presence of barium-impregnated silicone).

At least one glaucoma drainage implant, the ExPRESS miniature glaucoma shunt (Optonol Ltd.), is made from 316 L stainless steel. However, many other glaucoma drainage implants are made from nonmetallic materials and are safe for patients undergoing MRI procedures. Commonly used devices that do not contain metal include the following:

- Baerveldt glaucoma drainage implant (Pharmacia Co.),

- Krupin-Denver eye valve to disk implant (E. Benson Hood Laboratories),

- Ahmed glaucoma valve (New World Medical),

- Molteno drainage device (Molteno Ophthalmic Ltd.), and

- Joseph valve (Valve Implants Limited).

\section{Hemostatic (Ligating) Vascular Clips}

In general, it was previously believed that because virtually all hemostatic (also called ligating) vascular clips and similar devices (including "endoclips" deployed through endoscopes) are made from nonferromagnetic materials such as tantalum, titanium, and certain forms of stainless steel, patients with these implants are not at risk for injury in association with MRI procedures [11, 12, 14, 38]. Furthermore, patients with nonferromagnetic versions of these implants may undergo MRI examinations procedures immediately after they are placed. To date, for the hemostatic clips that have undergone MRI testing, there has been no patient injury or other problem related to MRI.
However, several new hemostatic clips in use today present potential problems for patients referred for MRI procedures. Patients with these clips require special attention to ensure the safe use of MRI. In some cases, MRI is deemed unsafe. In others, a waiting period is necessary and radiographs must be obtained and inspected to determine whether the clips are present before performing MRI. Specific MRI-related labeling statements for the hemostatic clips that require further attention during the MRI screening procedure are as follows:

Resolution Clip-The Resolution Clip (Boston Scientific) is indicated for placement in the gastrointestinal tract for endoscopic marking or hemostasis. Currently, the Resolution Clip is labeled, as follows [39]: "Do not perform MRI procedures on patients who have had clips placed within their gastrointestinal tract, as this could be harmful to patients."

Long Clip HX-600-090L-The Long Clip HX-600-090L (Olympus Medical Systems) is indicated for placement within the gastrointestinal tract for endoscopic marking, hemostasis, or closure of gastrointestinal tract luminal perforations within $20 \mathrm{~mm}$ as a supplementary method. Currently, the Long Clip HX-600-090L is labeled as follows: "Do not perform MRI procedures on patients who have clips placed within their gastrointestinal tracts. This could be harmful to the patient."

Additional information-Olympus endoscopic clips have been shown to remain in the patient an average of 9.4 days, but retention is based on a variety of factors and may result in a longer retention period. Before MRI, the physician should confirm that there are no residual clips in the gastrointestinal tract. The following techniques may be used for confirmation:

(1) View the lesion under radiologic imaging. Olympus clip-fixing devices are radiopaque. Using radiography, the physician can determine if any residual clips are in the gastrointestinal tract. If no clips are evident under radiologic imaging, MRI may be performed.

(2) Endoscopically examine the lesion. If no clips remain at the lesion, MRI may be performed.

QuickClip2, HX-201LR-135, and HX-201UR-135-The QuickClip2, HX-201LR-135, and HX-201UR-135 (Olympus Medical Systems) are indicated for placement within the gastrointestinal tract for endoscopic mark- 


\section{Screening Patients for MRI}

ing, hemostasis, or closure of gastrointestinal tract luminal perforations within $20 \mathrm{~mm}$ as a supplementary method. Currently, the QuickClip2 (HX-201LR-135 and HX-201UR-135) is labeled as follows: "Do not perform MRI procedures on patients who have clips placed within their gastrointestinal tracts. This could be harmful to the patient."

Additional information-Olympus endoscopic clips have been shown to remain in the patient an average of 9.4 days, but retention is based on a variety of factors and may result in a longer retention period. Before MRI, the physician should confirm that there are no residual clips in the gastrointestinal tract. The following techniques may be used for confirmation:

(1) View the lesion under radiologic imaging. Olympus clip-fixing devices are radiopaque. Using radiography, the physician can determine whether any residual clips are in the gastrointestinal tract. If no clips are evident under radiologic imaging, MRI may be performed.

(2) Endoscopically examine the lesion. If no clips remain at the lesion, MRI may be performed.

QuickClip2 Long, HX-201LR-135L, and HX-201UR-135L-The QuickClip2 Long, HX-201LR-135L, and HX-201UR-135L (Olympus Medical Systems) are indicated for placement within the gastrointestinal tract for endoscopic marking, hemostasis, or closure of gastrointestinal tract luminal perforations within $20 \mathrm{~mm}$ as a supplementary method. Currently, the QuickClip2 Long (HX-201LR-135L and HX-201UR$135 \mathrm{~L}$ ) is labeled as follows: "Do not perform MRI procedures on patients who have clips placed within their gastrointestinal tracts. This could be harmful to the patient."

Additional information-Olympus endoscopic clips have been shown to remain in the patient an average of 9.4 days, but retention is based on a variety of factors and may result in a longer retention period. Before MRI, the physician should confirm that there are no residual clips in the gastrointestinal tract. The following techniques may be used for confirmation:

(1) View the lesion under radiologic imaging. Olympus clip-fixing devices are radiopaque. Using radiography, the physician can determine whether any residual clips are in the gastrointestinal tract. If no clips are evident under radiologic imaging, MRI may

be performed.

(2) Endoscopically examine the lesion. If no clips remain at the lesion, MRI may be performed.

\section{PillCam (M2A) Capsule Endoscopy Device}

The PillCam (M2A) Capsule Endoscopy Device (Given Imaging) is an ingestible device for use in the gastrointestinal tract. Peristalsis moves the PillCam (M2A) Capsule smoothly and painlessly throughout the gastrointestinal tract, transmitting color video images as it passes. The procedure allows patients to continue daily activities during the endoscopic examination. The PillCam (M2A) Capsule Endoscopy Device has been used to diagnose diseases of the small intestine including Crohn's disease, celiac disease and other malabsorption disorders, benign and malignant tumors of the small intestine, vascular disorders, and medication-related small-bowel injuries.

MRI safety information-Undergoing MRI while the capsule is inside the patient's body may result in serious damage to his or her intestinal tract or abdominal cavity. If the patient did not positively verify the excretion of the PillCam (M2A) Capsule Device, he or she should contact the physician for evaluation and possible abdominal radiography before undergoing an MRI examination [12, 14].

\section{Sleuth Implantable ECG-Monitoring System}

The Sleuth Implantable ECG-Monitoring System (Sleuth IMD model 2010, Transoma Medical) is an implantable, patient-activated and automatically activated monitoring system that records subcutaneous ECG and is indicated for patients with clinical syndromes or at increased risk of cardiac arrhythmias and patients who experience transient symptoms that may suggest a cardiac arrhythmia [12, 14].

MRI information-This device is MR conditional at 1.5-T MRI. The monitoring system does not need to be adjusted during MR scanning. However, MR scanning may cause electromagnetic interference that may cause the system to record ECG events. MR scanning may also interfere with the quality of the ECG signal. Therefore, ECG data acquired during MRI may be inaccurate or unusable.

The Sleuth IMD model 2010 was determined to be MR conditional based on information provided in the following document published by the American Society for
Testing and Materials (ASTM) International (Designation F2503-05) Standard Practice for Marking Medical Devices and Other Items for Safety in the Magnetic Resonance Environment. ASTM International, 100 Barr Harbor Drive, PO Box C700, West Conshohocken, Pennsylvania, 2005 [25].

Nonclinical testing has shown that the Sleuth IMD is MR conditional. It can be scanned safely under the following conditions:

- static magnetic field of $1.5 \mathrm{~T}$,

- spatial gradient magnetic field of $250 \mathrm{G} /$ $\mathrm{cm}$, and

- maximum MR system-reported wholebody-averaged specific absorption rate (SAR) of $3.5 \mathrm{~W} / \mathrm{kg}$ for 15 minutes of scanning.

In nonclinical testing in a phantom, the device produced a temperature rise of $1.3^{\circ} \mathrm{C}$ or less at a maximum whole-body-averaged SAR of $3.5 \mathrm{~W} / \mathrm{kg}$ for 15 minutes of MR scanning in a 1.5-T MR system (Magnetom, Siemens Medical Solutions; software Numaris/4, Version Syngo MR 2002B DHHS active-shielded, horizontal field scanner, Siemens).

Instructions for use-The maximum whole-body-averaged SAR reported was measured by the MR system. MR image quality may be compromised if the area of interest is in the same area or is relatively close to the position of the Sleuth IMD. Table 1 provides maximum signal voids (artifact size) for the standard imaging pulse sequences at $1.5 \mathrm{~T}$ per ASTM F2119.

The effect of performing MRI procedures using higher static magnetic field or higher levels of RF energy on a patient with the Sleuth IMD has not been determined. MRI health care professionals are advised to contact Transoma Medical to ensure that the latest safety information is obtained and is carefully followed to ensure patient safety relative to the use of an MR procedure.

Note that magnetic forces may act on the housing of the implanted Sleuth IMD and result in a tugging sensation that patients may feel. This force does not pose a safety hazard, but to mitigate patient alarm, patients should be made aware of the possibility of such a sensation.

\section{VeriChip Microtransponder \\ The VeriChip Microtransponder (VeriChip Corporation) is a miniaturized, implant-}


TABLE I: Maximum Signal Voids (Artifact Size) for the Standard Imaging Pulse Sequences at I.5 T per American Society for Testing and Materials International (F2119)

\begin{tabular}{c|l|l}
\hline Signal Void $\left(\mathrm{cm}^{2}\right)$ & \multicolumn{1}{|c|}{ Pulse Sequence } & Imaging Plane \\
\hline 115 & T1-weighted spin echo & Parallel \\
68 & T1-weighted spin echo & Perpendicular \\
162 & Gradient echo & Parallel \\
280 & Gradient echo & Perpendicular \\
\hline
\end{tabular}

Note-Data apply to the Sleuth implantable ECG-monitoring system (Sleuth IMD model 2010, Transoma Medical).

able RF identification device. The microtransponder is a passive device that contains an electronic circuit that is activated externally by a low-power electromagnetic field emitted by a battery-powered scanner. The microtransponder is implanted subcutaneously. With regard to MRI procedures, the labeling for this device states: "Patients with the VeriChip Microtransponder may safely undergo MRI diagnostics, in up to 7-T cylindric systems" $[12,14]$.

Instructions for patients undergoing MRI-

- The patient should be monitored continuously throughout the MRI procedure using visual and audio means (e.g., intercom system).

- Instruct the patient to alert the MR system operator of any unusual sensations or problems so that, if necessary, the MR system operator can immediately terminate the procedure.

- Provide the patient with a means to alert the MR system operator of any unusual sensations or problems.

- Do not perform MRI if the patient is sedated, anesthetized, confused, or otherwise unable to communicate with the MR system operator.

\section{Insulin Pumps}

The MRI information pertains to the following insulin pumps from Animas Corporation, a Johnson \& Johnson Company:

- Animas 2020 Insulin Pump,

- IR Animas 1200,

- IR 1000 Insulin Pump,

- IR 1100 Insulin Pump, and

- IR 1200 Insulin Pump.

Each insulin pump indicated should not be exposed to very strong electromagnetic fields, such as those from MRI units, RF welders, or magnets used to pick up automobiles. Very strong magnetic fields, such as that associated with MRI, can "magnetize" the portion of the insulin pump's motor that regulates in- sulin delivery and, thus, damage this device.

For the patient: If you plan to undergo an MRI, remove the insulin pump beforehand and keep it outside of the MR system room during the procedure. If the pump is accidentally allowed into the MR system room, disconnect the pump immediately and contact Animas Pump Support for important instructions.

For the health care professional: Do not bring the insulin pump into the MR system at any time. If the pump is accidentally allowed into the MR system room, disconnect the pump immediately and contact Animas Pump Support for important instructions.

Cozmo Pump: an infusion pump-According to the User Manual for the Cozmo Pump (Deltec), which is a device used to administer insulin, the following is stated regarding MRI:

Caution: Avoid strong electromagnetic fields, like those present with Magnetic Resonance Imaging (MRI) and direct $\mathrm{x}$-ray, as they can affect how the pump works. If you cannot avoid them, you must take the pump off.

MiniMed 2007 Implantable Insulin Pump System-The MiniMed 2007 Implantable Insulin Pump System (Medtronic) is designed to withstand common electrostatic and electromagnetic interference but must be removed before an MR procedure. Any magnetic field exceeding $600 \mathrm{G}$ will interfere with the proper functioning of the pump for as long as the pump remains in that field. Fields higher than that, such as those emitted by an MR system, may cause irreparable damage to the pump.

By comparison, infusion sets (MMT11X, MMT-31X, MMT-32X, MMT-37X, MMT-39X; Medtronic) used with this device contain no metallic components and are safe to be used and can remain attached to the patient during an MR procedure. The only exceptions are Polyfin infusion sets. Polyfin infusion sets (MMT-106 and MMT-107,
MMT-16X, MMT-30X, MMT-36X; Medtronic) have a surgical steel needle that remains in the subcutaneous tissue. These infusion sets should be removed before any MR procedure.

\section{Codman Hakim Programmable Valve}

The Codman Hakim Programmable Valve (Codman, a Johnson \& Johnson Company) offers the ability to optimize the opening pressure of a CSF shunt system before and after implantation. This feature is considered to be an important one because the condition of the patient with the shunt will often change over the course of treatment. The use of a programmable CSF shunt valve allows the surgeon to noninvasively change the opening pressure, negating the need for revision surgery to alter the valve pressure. Furthermore, the programmability of the valve may allow the development of specialized treatment regimes.

The opening pressure of the Codman Hakim Programmable Valve is changed through the use of an externally applied magnetic field. The spring in the ball-andspring mechanism of the valve sits atop a rotating spiral cam that contains a stepper motor. Applying a specific magnetic field to the stepper motor will cause the cam to turn slightly, increasing or decreasing the tension on the spring and ball, thus changing the opening pressure of the valve.

With regard to MRI, the product insert for the Codman Hakim Programmable Valve states:

MRI information-The Codman Hakim Programmable Valve is considered MR Conditional according to ASTM F 2503. The valve demonstrates no known hazards when an MRI is performed under the following conditions:

- MRI can be performed at any time after implantation

- Use an MR system with a static magnetic field of $3 \mathrm{~T}$ or less

- Use an MR system with a spatial gradient of 720 gauss/cm or less

- Limit the exposure to RF energy to a whole-body-averaged specific absorption rate (SAR) of $3 \mathrm{~W} / \mathrm{kg}$ for 15 minutes

- Verify the valve setting after the MRI procedure (see Programming the Valve, product insert information provided with the valve)

In non-clinical testing, the valve produced a temperature rise of 0.4 degrees $\mathrm{C}$ at a max- 


\section{Screening Patients for MRI}

imum whole-body-averaged specific absorption rate $(\mathrm{SAR})$ of $3.0 \mathrm{~W} / \mathrm{kg}$ for 15 minutes of MR scanning in a 3-T Excite, General Electric MR scanner.

MR image quality may be compromised if the area of interest is relatively close to the device. Distortion may be seen at the boundaries of the artifact. Therefore, optimization of the MR imaging parameters may be necessary. The following chart provides a comparison between the signal void and imaging pulse sequence at 3-Tesla:

\begin{tabular}{c|l|l}
\hline $\begin{array}{c}\text { Signal Void } \\
\text { Size }\end{array}$ & \multicolumn{1}{|c|}{$\begin{array}{c}\text { Pulse } \\
\text { Sequence }\end{array}$} & $\begin{array}{c}\text { Scan Orientation } \\
\text { Relative to Implant }\end{array}$ \\
\hline $1590 \mathrm{~mm}^{2}$ & T1-SE & long axis view \\
$1022 \mathrm{~mm}^{2}$ & T1-SE & short axis view \\
$2439 \mathrm{~mm}^{2}$ & GRE & long axis view \\
$2404 \mathrm{~mm}^{2}$ & GRE & short axis view \\
\hline
\end{tabular}

\section{Summary}

To prevent incidents and accidents, it is vital to be cognizant of basic information as well as the latest findings that impact the use of MRI to ensure safety for patients, staff members, and others. Having this knowledge is particularly important because of the evolutionary advancements in MRI technology and the increased potential for hazardous situations to occur in this environment.

\section{References}

1. Shellock FG, Crues JV 3rd. MR procedures: biologic effects, safety, and patient care. Radiology 2004; 232:635-652

2. Shellock FG. Biomedical implants and devices: assessment of magnetic field interactions with a 3.0-Tesla MR system. J Magn Reson Imaging 2002; 16:721-732

3. Schenck JF. Safety of strong, static magnetic fields. J Magn Reson Imaging 2000; 12:2-19

4. Schaefer DJ, Bourland JD, Nyenhuis JA. Review of patient safety in time-varying gradient fields. $J$ Magn Reson Imaging 2000; 12:20-29

5. Shellock FG. Radiofrequency energy-induced heating during MR procedures: a review. J Magn Reson Imaging 2000; 12:30-36

6. McJury M, Shellock FG. Auditory noise associated with MR procedures: a review. J Magn Reson Imaging 2000; 12:37-45

7. Shellock FG, Kanal E. Policies, guidelines, and recommendations for MR imaging safety and patient management. SMRI Safety Committee. $J$ Magn Reson Imaging 1991; 1:97-101

8. Kanal E, Shellock FG. Policies, guidelines, and recommendations for MR imaging safety and patient management. SMRI Safety Committee. J
Magn Reson Imaging 1992; 2:247-248

9. Shellock FG, Kanal E. Guidelines and recommendations for MR imaging safety and patient management. III. Questionnaire for screening patients before MR procedures. The SMRI Safety Committee. J Magn Reson Imaging 1994; 4:749751

10. Wood TO. MRI safety. In: Akay M, ed. Wiley encyclopedia of biomedical engineering. Hoboken, NJ: Wiley, 2006:2360-2371

11. Shellock FG. Chapter 24: Magnetic resonance bioeffects, safety, and patient management. In: Edelman R, Hesselink J, Zlatkin M, Crues JV 3rd, eds. Clinical magnetic resonance imaging, 3rd ed. Philadelphia, PA: Saunders, 2005:680733

12. Shellock FG. Reference manual for magnetic resonance safety, implants, and devices: 2008 edition. Los Angeles, CA: Biomedical Research Publishing Group, 2008

13. Shellock FG. Magnetic resonance procedure: health effects and safety. Boca Raton, FL: CRC Press, 2001

14. Shellock FG. MRIsafety.com: MRI safety, bioeffects and patient management. www.mrisafety. com. Published 2001. Updated 2008. Accessed June 25, 2008

15. Website for the Institute for Magnetic Resonance Safety, Education, and Research. www.IMRSER. org. Published 2001-2004. Updated 2008. Accessed 25, 2008

16. Kanal E, Barkovich AJ, Bell C, et al.; ACR Blue Ribbon Panel on MR Safety. ACR guidance document for safe MR practices: 2007. AJR 2007; 188:1447-1474

17. Sawyer-Glover A, Shellock FG. Pre-MRI procedure screening: recommendations and safety considerations for biomedical implants and devices. J Magn Reson Imaging 2000; 12:92-106

18. Kuhl CK, Träber F, Schild HH. Whole-body highfield-strength (3.0-T) MR imaging in clinical practice. I. Technical considerations and clinical applications. Radiology 2008; 246:675-696

19. Kuhl CK, Träber F, Gieseke J, et al. Whole-body high-field-strength (3.0-T) MR imaging in clinical practice. II. Technical considerations and clinical applications. Radiology 2008; 247: 1635

20. Levine GN, Gomes AS, Arai AE, et al.; American Heart Association Committee on Diagnostic and Interventional Cardiac Catheterization; American Heart Association Council on Clinical Cardiology; American Heart Association Council on Cardiovascular Radiology and Intervention. Safety of magnetic resonance imaging in patients with cardiovascular devices: an American Heart Association scientific statement from the Committee on Diagnostic and Interventional
Cardiac Catheterization, Council on Clinical Cardiology, and the Council on Cardiovascular Radiology and Intervention: endorsed by the American College of Cardiology Foundation, the North American Society for Cardiac Imaging, and the Society for Cardiovascular Magnetic Resonance. Circulation 2007; 116:2878-2891

21. Shinbane J, Colletti P, Shellock FG. MR in patients with pacemakers and ICDs: defining the issues. J Cardiovasc Magn Reson 2007; 9:5-13

22. Baker KB, Tkach JA, Nyenhuis JA, et al. Evaluation of specific absorption rate as a dosimeter of MRI-related implant heating. J Magn Reson Imaging 2004; 20:315-320

23. Shellock FG. Comments on MRI heating tests of critical implants. (editorial) J Magn Reson Imaging 2007; 26:1182-1185

24. Woods TO. Standards for medical devices in MRI: present and future. J Magn Reson Imaging 2007; 26:1186-1189

25. American Society for Testing and Materials (ASTM) International. Standard practice for marking medical devices and other items for safety in the magnetic resonance environment. West Conshohocken, PA: ASTM International, 2005; designation: F2503-05

26. The Joint Commission Website. Sentinel event alert: preventing accidents and injuries in the MRI suite. www.jointcommission.org/SentinelEvents/SentinelEventAlert/sea_38.htm.

Published February 14, 2008. Accessed June 25, 2008

27. U.S. Food and Drug Administration, Center for Devices and Radiological Health Website. A primer on medical device interactions with magnetic resonance imaging systems. www.fda.gov/ cdrh/ode/primerf6.html. Published February 7, 1997. Updated May 23, 1997

28. Shellock FG, Crues JV 3rd. MR safety and the American College of Radiology White Paper. AJR 2002; 178:1349-1352

29. Armstrong ML, Elkins L. Body art and MRI. Am J Nurs 2005; 105:65-66

30. DeBoer S, Fishman D, Chwals W, Straus C, Amundson T. Body piercing/tattooing and trauma diagnostic imaging: medical myths vs realities. $J$ Trauma Nurs 2007; 14:35-38

31. Muensterer OJ. Temporary removal of navel piercing jewelry for surgery and imaging studies. Pediatrics 2004; 114:e384-e386

32. Ceballos EM, Parrish RK. Plain film imaging of Baerveldt glaucoma drainage implants. Am J Neuroradiol 2002; 23:935-937

33. Dahan E, Carmichael TR. Implantation of a miniature glaucoma device under a scleral flap. $J$ Glaucoma 2005; 14:98-102

34. Hong CH, Arosemena A, Zurakowski D, Ayyala RS. Glaucoma drainage devices: a systematic 


\section{Shellock and Spinazzi}

literature review and current controversies. Surv Ophthalmol 2005; 50:48-60

35. Jeon TY, Kim HJ, Kim ST, Chung TY, Kee C. MR imaging features of giant reservoir formation in the orbit: an unusual complication of Ahmed glaucoma valve implantation. Am J Neuroradiol 2007; 28:1565-1566
36. Sidoti PA, Baerveldt G. Glaucoma drainage implants. Curr Opin Ophthalmol 1994; 5:85-98

37. Traverso CE, De Feo F, Messas-Kaplan A, et al. Long term effect on IOP of a stainless steel glaucoma drainage implant (Ex-PRESS) in combined surgery with phacoemulsification. $\mathrm{Br} J$ Ophthalmol 2005; 89:425-429 [Erratum in BrJ
Ophthalmol 2005; 89:645]

38. Raju GS, Gajula L. Endoclips for GI endoscopy. Gastrointest Endosc 2004; 59:267-279

39. Boston Scientific Website. Resolution Clip information. www.bostonscientific.com/templatedata/ imports/collateral/Endoscopy/prospec_resolution_01_us.pdf. Accessed June 25, 2008

\section{FOR YOUR INFORMATION}

The reader's attention is directed to the commentary on this article, which appears on the following pages.

\section{FOR YOUR INFORMATION}

The reader's attention is directed to part I accompanying this article, titled "MRI Safety Update 2008: Part I, MRI Contrast Agents and Nephrogenic Systemic Fibrosis," which begins on page xxx. 